\title{
Human sex hormone-binding globulin variants associated with hyperandrogenism and ovarian dysfunction
}

\author{
Kevin N. Hogeveen, ${ }^{1}$ Patrice Cousin, ${ }^{2}$ Michel Pugeat, ${ }^{2}$ Didier Dewailly, ${ }^{3}$ Benoît Soudan, ${ }^{3}$ \\ and Geoffrey L. Hammond ${ }^{1}$ \\ ${ }^{1}$ Department of Obstetrics and Gynecology; Department of Pharmacology and Toxicology; \\ and Canadian Institutes of Health Research Group in Fetal and Neonatal Health and Development; \\ University of Western Ontario, London, Ontario, Canada \\ ${ }^{2}$ Laboratoire de la Fédération d'Endocrinologie, Hôpital de l'Antiquaille, Hospices Civils de Lyon and \\ Institut National de la Santé et de la Recherche Médicale, U 329, Lyon, France \\ ${ }^{3}$ Service d'Endocrinologie et de Médecine de la Reproduction, Centre Hospitalier et Universitaire de Lille, Lille, France \\ Address correspondence to: Geoffrey L. Hammond, London Regional Cancer Centre, 790 Commissioners Road East, \\ London, Ontario N6A 4L6, Canada. Phone: (519) 685-8637; Fax: (519) 685-8616; E-mail: ghammond@uwo.ca. \\ Kevin N. Hogeveen and Patrice Cousin contributed equally to this work. \\ Received for publication August 24, 2001, and accepted in revised form February 18, 2002.
}

The access of testosterone and estradiol to target tissues is regulated by sex hormone-binding globulin (SHBG) in human blood. Serum SHBG levels are low in patients with hyperandrogenism, especially in association with polycystic ovarian syndrome (PCOS) and in individuals at risk for diabetes and heart disease. Here, we identify $S H B G$ coding region variations from a compound heterozygous patient who presented with severe hyperandrogenism during pregnancy. Serum SHBG levels in this patient measured 2 years after her pregnancy were exceptionally low, and her non-protein-bound testosterone concentrations greatly exceeded the normal reference range. A single-nucleotide polymorphism within the proband's maternally derived $S H B G$ allele encodes a missense mutation, P156L, which allows for normal steroid ligand binding but causes abnormal glycosylation and inefficient secretion of SHBG. This polymorphism was identified in four other patients with either PCOS, ioiopathic hirsutism, or ovarian failure. The proband's paternal $S H B G$ allele carries a single-nucleotide deletion within exon 8, producing a reading-frame shift within the codon for E326 and a premature termination codon. $\mathrm{CHO}$ cells transfected with a SHBG cDNA carrying this mutation fail to secrete the predicted truncated form of SHBG. To our knowledge, these are the first examples of human $S H B G$ variants linked to hyperandrogenism and ovarian dysfunction.

J. Clin. Invest. 109:973-981 (2002). DOI:10.1172/JCI200214060.

\section{Introduction}

Human sex hormone-binding globulin (SHBG) transports testosterone and estradiol in the blood $(1,2)$. Blood concentrations of SHBG are a major determinant of the metabolic clearance of these sex steroids and their access to target tissues (3), and their measurements provide a means of estimating the amounts of circulating non-protein-bound, or "free," sex steroids (4). Abnormally low serum SHBG levels are frequently found in women with polycystic ovarian syndrome (PCOS) and contribute to hyperandrogenic symptoms such as hirsutism and acne (1). Serum SHBG levels are also reduced in patients with type 2 diabetes and coronary heart disease $(5,6)$. The reason that SHBG levels are low in serum samples from many of these individuals is unclear, but it has been reported that SHBG deficiencies are inherited $(7,8)$.

Human SHBG is a homodimeric plasma glycoprotein encoded by a $4-\mathrm{kb}$ gene spanning eight exons on the short arm (p12 $\rightarrow$ p13) of chromosome $17(9,10)$. It is produced by hepatocytes $(11,12)$ under the control of various hormonal and metabolic regulators $(13,14)$. Exon 1 of human $S H B G$ contains the coding sequence for the secretion signal polypeptide, while exons 2-8 encode two contiguous laminin G-like (LG) domains $(9,15)$. The amino-terminal LG domain encoded by exons 2-4 contains the steroid-binding site (15), the dimer interface (16), and several cation-binding sites (17). The functional significance of the carboxy-terminal LG domain of SHBG is less well established, but removal of only a few amino acids from the carboxy-terminus of the rat orthologue of human SHBG, which is generally known as the androgen-binding protein (ABP), prevents secretion of a functional protein when expressed in mammalian cells (18). This domain also normally contains two sites for $\mathrm{N}$-glycosylation, one of which is invariably conserved in SHBG molecules across a wide range of mammalian species (19).

A single-nucleotide polymorphism (SNP) within the human $S H B G$ coding sequence is responsible for a common electrophoretic variant with an additional $\mathrm{N}$-glycosylation consensus sequence within the carboxyterminal LG domain (20). An additional carbohydrate chain at this position has no impact on the steroidbinding activity of SHBG, but it may reduce the plasma clearance of SHBG and account for a modest accumu- 
lation of the variant protein in the blood (21). More recently, we have identified a polymorphic (TAAAA) repeat in the human $S H B G$ promoter that influences its transcriptional activity (22), but it remains to be determined whether this pentanucleotide repeat contributes to the differences in plasma SHBG levels between individuals. In this report, we have identified two sequence variations in the coding region of human $S H B G$ that account for unusually low serum SHBG levels in a patient with severe hyperandrogenism. Furthermore, at least one of these sequence variations is associated with hyperandrogenic states and ovarian dysfunction in several other patients.

\section{Methods}

Patients and clinical samples. The proband was a 27 -yearold patient from the north of France. Her body weight was normal, and she had a history of regular menstrual cycles. She reported a mild hirsutism that increased dramatically during pregnancy, and this was associated with other symptoms of virilism including deepening of the voice and enlargement of the clitoris. Her pregnancy was otherwise normal, and she delivered twin girls with no signs of virilization. Two years after this pregnancy, hirsutism was scored between 14 and 16 according to the scoring system of Ferriman and Gallwey (23), but other symptoms of virilism were no longer apparent. Blood samples were taken at this time, and serum total testosterone, androstenedione, 17-hydroxyprogesterone, and dehydro-epiandrosterone (DHEAS) levels were within the ranges for normal women. In contrast, serum SHBG was undetectable using a routine immunoassay. She was then placed under treatment with $50 \mathrm{mg}$ cyproterone acetate and $2 \mathrm{mg}$ estradiol taken orally 21 days each month. Under this treatment, additional blood samples were taken for more detailed studies of serum SHBG levels and for genetic analysis. Blood samples were also taken from the proband's father and sister for DNA sequence analysis and serum SHBG measurements. At the time of blood sampling, her father was under treatment for hypertension and her sister was taking an oral contraceptive containing $20 \mu \mathrm{g}$ ethinyl estradiol and $150 \mu$ g gestodene.

Blood samples were also taken from the following groups of women for DNA sequence analysis and serum SHBG measurements: group I, 88 nonobese fertile women with regular menstrual cycles (i.e., normal controls); group II, 132 normal-weight women (body mass index $<25 \mathrm{~kg} / \mathrm{m}^{2}$ ) with symptoms of hirsutism, as defined by Ferriman and Gallwey (23); group III, 93 overweight women (body mass index $>25 \mathrm{~kg} / \mathrm{m}^{2}$ ) with symptoms of hirsutism; group IV, 69 women with no evidence of androgen excess, but with irregular menstrual cycles due to a variety of clinical problems including obesity, anorexia nervosa, precocious menopause, and hyperprolactinemia; group V, 53 women with 21-hydroxylase deficiency; group VI, 47 women with a variety of other endocrine disorders. Approximately $5 \%$ of the controls and patients studied were of North African origin, while the remainder were French Caucasian.

SHBG and testosterone assays. Two different immunoradiometric assays (SHBG-RIACT kit from CIS Bio International, Gif-sur-Yvette, France; and SHBG IRMA kit from Orion Diagnostica, Oulunsalo, Finland) were used to determine SHBG concentrations in serum samples, and these gave similar results. The values reported are those obtained using the SHBG-RIACT kit. The method used to measure the very low steroid-binding capacity of SHBG in the proband serum, and its binding affinity by Scatchard analysis, was based on an established ligand-binding assay (24) except that an anti-SHBG mAb-Sepharose (kindly provided by Catherine Grenot, Institut National de la Santé et de la Recherche Médicale, U 329) was used instead of concanavalin A-Sepharose. A conventional steroid-binding capacity assay (25) and a time-resolved immunofluorometric assay (26) were also used to measure SHBG levels in serum samples and cell culture medium. Western blotting was used to examine the electrophoretic properties of SHBG in serum (21), as well as in culture medium samples and cell extracts (27). In order to obtain sufficient SHBG from the proband serum for Western blot analysis, the SHBG in serum samples was first isolated by immunoaffinity adsorption (21).

Total serum testosterone levels (normal female reference range: $0.42-1.49 \mathrm{nM}$ ) and the amounts of non-SHBG-bound testosterone (normal female reference range: $62-236 \mathrm{pM}$ ) were determined in serum samples by radioimmunoassay (28). The serum distribution of testosterone was measured using the centrifugal ultrafiltration/dialysis method (3).

Screening for SHBG sequence variations. Two primer pairs were used to amplify the entire coding sequence for the SHBG precursor polypeptide from the proband's genomic DNA. The 5' amplicon primer set comprised a $5^{\prime}$ untranslated region-specific oligonucleotide sequence, $5^{\prime}$-GAGTTGTCTGAGCCGCCG-3'; and an oligonucleotide complementary to an intron 4 sequence, $5^{\prime}$-AGCCACCCAGCAGTGCTT- 3 '. The $3^{\prime}$ amplicon primer set comprised an intron 3-specific oligonucleotide, 5'-ACAGGAAGGTGGCAGAAA-3'; and an oligonucleotide complementary to a region $3^{\prime}$ to the polyadenylation site in exon $8,5^{\prime}$-GCCTGGTACATTGCTAG- $3^{\prime}$. The PCR products were inserted in the PCRBlunt II-TOPO vector from Invitrogen Life Technologies (Invitrogen Canada Inc., Burlington, Ontario, Canada) and sequenced. Exon sequences that deviated from the consensus SHBG sequence (GenBank accession no. M31651) were reamplified in a PCR using exon-specific primer pairs (20) and sequenced.

Expression of SHBG in Chinese hamster ovary cells. We used a pRc/CMV vector (Invitrogen Life Technologies) to express wild-type and mutated SHBG cDNAs in Chinese hamster ovary (CHO) cells, as described previously (27). At near confluence, the transfected cells were washed with PBS and cultured for a further 2 days in serum-free DMEM (Invitrogen Life Technologies). The 
SHBG content of cell culture medium was examined by Western blot analysis (27). In addition, we measured the SHBG content of the CHO cells grown in this way by Western blotting. To accomplish this, soluble proteins were extracted from the $\mathrm{CHO}$ cells by homogenization in $40 \mathrm{mM}$ Tris, pH 7.5, $1 \mathrm{mM}$ EDTA, $150 \mathrm{mM}$ $\mathrm{NaCl}$, while insoluble protein was extracted from the resulting pellet using $10 \mathrm{mM}$ Tris, $\mathrm{pH} 8,100 \mathrm{mM}$ EDTA containing $0.5 \%$ sodium-dodecyl-sulphate.

Consensus and variant $S H B G$ alleles were also inserted as 4-kbp HindIII-XbaI genomic DNA cassettes in $\mathrm{pRc} / \mathrm{CMV}$. The HindIII site was introduced within the $5^{\prime}$ untranslated sequence $36 \mathrm{bp}$ from the translation initiation codon in exon 1 , while the $\mathrm{XbaI}$ site was introduced after $68 \mathrm{bp}$ of noncoding sequence $3^{\prime}$ from the stop codon within exon 8 . These constructs were transfected into mouse BW-1 hepatoma cells (29) and human HepG2 hepatoblastoma cells using LIPOFECTOMINE, as recommended by Invitrogen Life Technologies, and pools of stable transformants were obtained by culture under G418 selection (27). Cell culture medium was harvested from near confluent cultures grown in serum-free medium for 2 days for Western blotting. In addition, the transfected BW-1 cells were also harvested from near-confluent cultures grown in $\alpha M E M$ containing $10 \%$ FBS for RNA extraction using TRIzol solution (Invitrogen Life Technologies).The RNA obtained in this way was used for RT-PCR and Northern blot (30) analyses of SHBG transcripts. The primer sequences used for the RT-PCR analyses were as follows: exon 3 forward primer, $5^{\prime}$-GACCCAGAGGGAGTGATTT- $3^{\prime}$; exon 4 forward primer, $5^{\prime}$ GAGGGGGACTCTGTG-3'; exon 5 reverse primer, $5^{\prime}$ GAATTCTGCCTGAGT-3'.

In addition, the rates of SHBG secretion from pools of BW-1 cells that constitutively express the MspI variant or consensus alleles were assessed by growing replicate $(n=3)$ cultures to near confluence, and then removing the culture medium, washing the cells once with PBS, and replacing the medium. Samples of culture medium were then removed at timed intervals over 6 hours for SHBG measurements using an ultrasensitive, time-resolved immunofluorometric assay (26). At the end of this experiment, cells were harvested for RNA extraction to assess the relative amounts of $S H B G$ transcripts by Northern blotting (30).

\section{Results}

Identification of two different coding sequence variations within the $S H B G$ alleles of a single individual. We measured the proband's serum SHBG levels by two different immunoassays and conventional steroid-binding capacity assays. In each case, the values obtained (0.5-1 nmol/l) were approximately an order of magnitude lower than those found in other hyperandrogenic patients, and about 50 times lower than those in normal age-matched women $(30-69 \mathrm{nmol} / \mathrm{l})$. These results were even more remarkable because the proband was being treated at the time of blood sampling with an oral estrogen and antiandrogen formulation that would normally be expected to increase serum SHBG levels (see Methods). Overlapping genomic fragments spanning the entire transcription unit encoding SHBG $(9,12)$ were therefore PCR-amplified using the proband's DNA (Figure 1a). Sequence analysis of the PCR products revealed two alleles based on the presence or absence of an SNP $\left(\mathrm{P}_{1}\right)$ within exon 4 (Figure $1 \mathrm{~b})$. This occurs on one allele (Figure $1 \mathrm{~b}$ ) and converts the codon (CCG) for P156 into a leucine codon (CTG). On the other allele, there is a single-nucleotide deletion $\left(\mathrm{P}_{2}\right)$ within exon 8 (Figure $1 \mathrm{~b}$ ). The transcription units of both $S H B G$ alleles were completely sequenced, and no other deviations from the published human $S H B G$ sequences $(9,31)$ were noted. This analysis also included the 800-bp SHBG promoter sequence (GenBank accession no. M31651).

Screening for the MspI polymorphism. The SNP within exon 4 disrupts an MspI site (CCGG) at the boundary between exon 4 and intron 4 (CC/TGGT, intron splice junction underlined) in the consensus $S H B G$ sequence (9). We therefore established a PCR-based assay to screen for this MspI polymorphism within the proband's family, as well as in 88 healthy control women and several groups of patients with various reproductive and/or endocrine disorders (see Table 1). The proband's father and sister do not carry the MspI polymorphism (Figure 2), and this allowed us to deduce that her deceased mother was a heterozygous carrier of this allele (see also below). The MspI polymorphism was not present in any of the control subjects tested, but four other heterozygous carriers of this

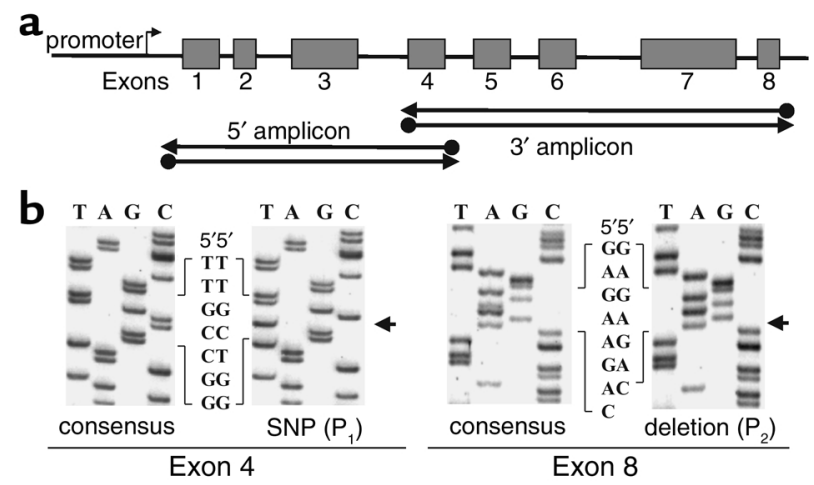

\section{Figure 1}

Identification of SHBG sequence variations in a DNA sample from the proband. (a) Schematic representation of the 4.3-kb genomic fragment comprising the human SHBG transcription unit showing the transcription start site (arrow), and the positions of exons in relation to the two overlapping amplicons generated for sequence analysis. (b) The sequence variations in exons 4 and 8 within separate $S H B G$ alleles from the proband are compared with the consensus SHBG sequences on the proband's other allele. The SNP in exon 4 is designated as $\mathrm{P}_{1}$, while the single-nucleotide deletion in exon 8 is designated as $\mathrm{P}_{2}$. Separate alleles were identified based on the presence or absence of $\mathrm{P}_{1}$ within the exon 4 sequences of the overlapping $5^{\prime}$ and $3^{\prime}$ amplicons shown in a. Arrows indicate the positions of sequence variations. 


\section{Table 1}

Details of normal women and various patient groups screened for the Mspl polymorphism

\begin{tabular}{lcccc} 
Group & $n$ & $\begin{array}{c}\text { BMI } \\
\left(\mathrm{kg} / \mathrm{m}^{2}\right)\end{array}$ & $\begin{array}{c}\text { SHBG } \\
(\mathrm{nmol} / \mathrm{I})\end{array}$ & $\begin{array}{c}\text { Mspl } \\
\text { polymorphism }\end{array}$ \\
I. normal women & 88 & $21.1 \pm 2.1$ & $55 \pm 29$ & 0 \\
II. nonobese/hirsute & 132 & $21.3 \pm 2.0$ & $39 \pm 22$ & 3 \\
III. obese/hirsute & 93 & $31.8 \pm 5.9$ & $25 \pm 17$ & 0 \\
IV. ovarian dysfunction & 69 & $28.0 \pm 8.7$ & $37 \pm 30$ & 1 \\
V. 21-hydroxylase deficiency & 53 & $24.6 \pm 6.8$ & $39 \pm 17$ & 0 \\
VI. endocrine diseases & 47 & $24.3 \pm 5.2$ & $42 \pm 24$ & 0 \\
\hline
\end{tabular}

Shown are numbers of subjects in each group screened, mean \pm SD body mass index (BMI) and serum SHBG levels, and number of Mspl polymorphism carriers identified.

MspI polymorphism were identified among the patients tested, and these individuals were French Caucasians. Three of them were within group II (nonobese/hirsute), two of whom had PCOS (Figure 2, patients $\mathrm{CV}$ and DC) and one of whom had idiopathic hirsutism and was taking l-thyroxine $(150 \mu \mathrm{g}$ daily) after a surgically removed thyroid cancer (not shown). The other carrier of this MspI polymorphism (Figure 2, patient CS) was identified within a group of women with ovarian dysfunction (Table 1, group IV). Her body mass index was normal, but she suffered from a precocious menopause at age 19 .

Both PCOS patients with the MspI polymorphism in group II were of normal weight and had serum SHBG levels $(17-27 \mathrm{nmol} / \mathrm{L})$ that were below the normal female reference range $(30-69 \mathrm{nmol} / \mathrm{l})$. The serum SHBG level $(35 \mathrm{nmol} / \mathrm{l})$ in the other patient from group II was in the low normal range, but this is a low level considering that she was under treatment with thyroid hormone (1). The two patients with PCOS had total serum testosterone (1.75 $\mathrm{nM}$ for patient CV and $1.90 \mathrm{nM}$ for patient DC) and non-SHBG-bound testosterone levels (288 pM for patient $\mathrm{CV}$ and $576 \mathrm{pM}$ for patient $\mathrm{DC}$ ) that were above the normal range. Although the serum testosterone measurements in the two other patients with an MspI polymorphism were within normal ranges (see Methods), these values may have been influenced by their unusual clinical conditions and/or treatments.

Screening for the single-nucleotide deletion in exon 8 . The single-nucleotide deletion $\left(\mathrm{P}_{2}\right)$ within exon 8 causes a frameshift within the codon for E326 and introduces a novel carboxyl-terminal coding sequence (S-L-P-P-L-FA) followed by a stop codon (Figure 3a). Although this results in the loss of a $B b s$ I site, the development of a screening assay was complicated because this occurs in close proximity to a relatively common $\mathrm{SNP}\left(\mathrm{P}_{3}\right)$, which introduces an additional consensus site for $\mathrm{N}$-glycosylation (Figure 3a) due to a D327N substitution (20). Nevertheless, discrimination between $\mathrm{P}_{3}$ and $\mathrm{P}_{2}$ could be accomplished by separate digestions of PCR products spanning this region with BbsI and Hinfl (Figure $3 b$ ). In subjects with either the $\mathrm{P}_{2}$ or $\mathrm{P}_{3}$ sequence variations, a $B b s I$ site in the consensus $S H B G$ sequence is disrupted (Figure $3 \mathrm{~b}$ ). However, alleles containing $\mathrm{P}_{3}$ also lack the
Hinfl site present in the consensus sequence, which is preserved in the allele containing $\mathrm{P}_{2}$ (Figure $3 \mathrm{~b}$ ).

This analysis demonstrated that the proband and her sister inherited the single-nucleotide deletion $\left(\mathrm{P}_{2}\right)$ in exon 8 from their father (Figure $3 \mathrm{~b}$ ) and confirms that the proband's $S H B G$ allele containing the MspI polymorphism was inherited from her deceased mother. We failed to identify other carriers of $\mathrm{P}_{2}$ in the 482 women (Table 1) who had been tested for the MspI polymorphism. Therefore, although $\mathrm{P}_{2}$ is clearly transmitted within the pedigree we have examined, it remains to be determined whether it exists within other patient groups characterized by low plasma levels of SHBG, or in other ethnic groups.

Biochemical properties of the proband's SHBG and its influence on the serum distribution of testosterone. The small amount of SHBG in the proband's serum was semipurified by immunoaffinity adsorption, and a Western blot indicated that the molecular sizes of its major electrophoretic isoforms are essentially normal (Figure 4a). However, it lacks a minor electrophoretic isoform (see bold arrow in Figure 4a) that corresponds to SHBG monomers in which the $\mathrm{N}$-glycosylation sites are partially utilized (32).

The steroid-binding properties of the SHBG isolated from the proband's serum were also examined by Scatchard analysis and were very similar to SHBG in normal male and female serum samples (Figure $4 \mathrm{~b}$ ). Thus, the SHBG in the proband's blood is capable of binding steroid, and we assessed its impact on the serum distribution of testosterone (Figure 4c). This indicated that even the small amount of SHBG in the proband's blood exerts some influence on the serum distribution of testosterone (Figure 4c). However, the percentage of non-protein-bound, or "free," testos-

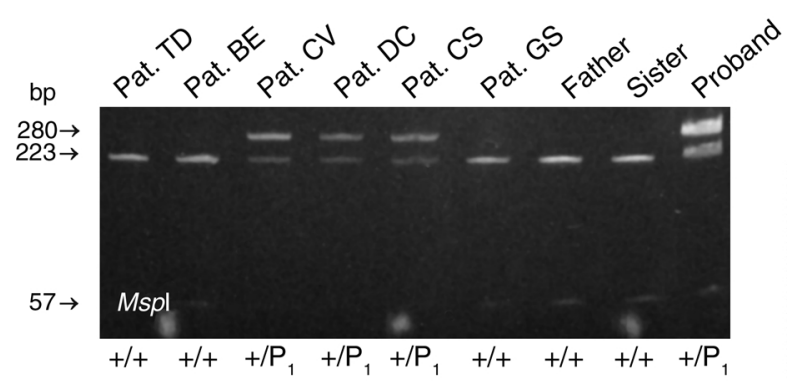

Figure 2

Identification of an SNP $\left(\mathrm{P}_{1}\right)$ that disrupts an Mspl site within the exon 4 /intron 4 boundary of human SHBG in DNA samples from the proband and female patients with reproductive disorders. Ethidium bromide-stained polyacrylamide electrophoresis gel of PCR-amplified exon 4 sequences digested with Mspl. The enzyme fails to completely digest PCR products from the variant allele in samples from the proband and three other patients (patients CV, DC, and CS). By contrast, PCR products (sizes, in bp, shown on the left) from the proband's father and sister and three patients (patients TD, BE, and GS) are digested completely by Mspl. Genotypes are: homozygous consensus sequence $(+/+)$ and heterozygous carriers of the Mspl SNP $\left(+/ \mathrm{P}_{1}\right)$. 
a $\ldots \mathrm{Gly}^{325} \mathrm{Glu}^{326} \mathrm{Asp}^{327} \mathrm{Ser}^{328} \mathrm{Ser}^{329} \mathrm{Thr}^{330} \mathrm{Ser}^{331} \mathrm{Phe}^{332} \mathrm{Cys}^{333} \mathrm{Leu}^{334} \ldots$ ... GGA GAA GAC TCT TCC ACC TCT TTT TGC CTG ... Bbsl $\frac{\text { Hinfl }}{\text { consensus sequence }}$

... Gly ${ }^{325} \mathrm{Glu}^{326} \mathrm{Ser}^{327} \mathrm{Leu}^{328} \mathrm{Pro}^{329} \mathrm{Pro}^{330} \mathrm{Leu}^{331} \mathrm{Phe}^{332} \mathrm{Ala}^{333}$ Stop

... GGA GAG ACT CTT CCA CCT CTT TTT GCC TGA Hinfl exon 8 deletion $\left(\mathrm{P}_{2}\right)$

... Gly ${ }^{325} \mathrm{Glu}^{326} \mathrm{Asn}^{327} \operatorname{Ser}^{328} \operatorname{Ser}^{329} \mathrm{Thr}^{330} \operatorname{Ser}^{331} \mathrm{Phe}^{332} \mathrm{Cys}^{333} \mathrm{Leu}^{334} \ldots$ ... GGA GAA AAC TCT TCC ACC TCT TTT TGC CTG ... D327N variant $\left(\mathrm{P}_{3}\right)$
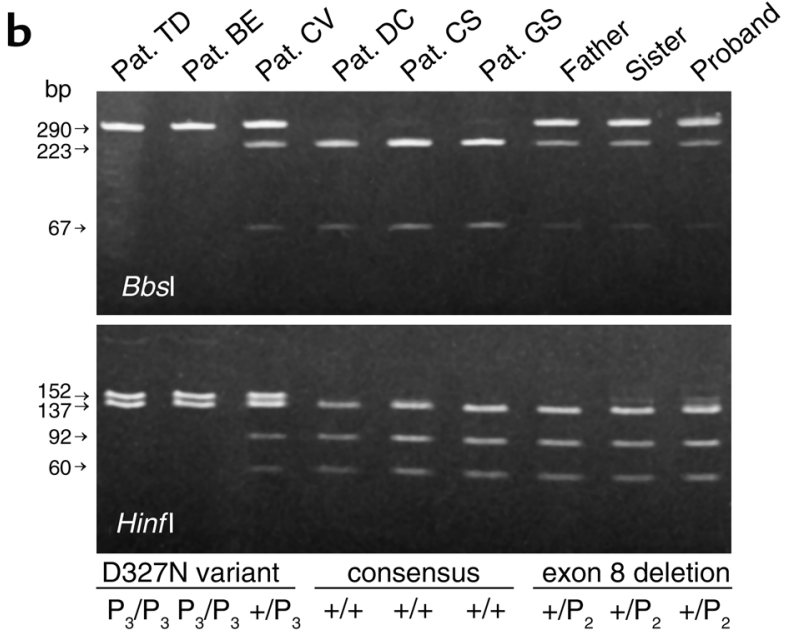

\section{Figure 3}

Identification of a single-nucleotide deletion $\left(\mathrm{P}_{2}\right)$ in exon 8 of the human $S H B G$ in DNA samples from the proband and her family members. (a) $\mathrm{P}_{2}$ disrupts a $B b s \mid$ site in the consensus $S H B G$ sequence and causes a frameshift in codon $\mathrm{E} 326$ that introduces a novel sequence followed by a stop codon. A common SNP $\left(\mathrm{P}_{3}\right)$ also disrupts this $B b s$ site, as well as a Hinfl site that is preserved in $\mathrm{P}_{2}$. The solid oval indicates the position of an additional $\mathrm{N}$-glycosylation site introduced by the $\mathrm{D} 327 \mathrm{~N}$ substitution caused by $\mathrm{P}_{3}$. (b) Ethidium bromide-stained polyacrylamide electrophoresis gel of PCR-amplified exon 8 sequences digested with either Bbsl or Hinfl. Approximately half of the PCR products from the proband and from her father and sister are resistant to Bbsl digestion but are completely digested with Hinfl. By contrast, patients who are homozygous (patients TD and BE) or heterozygous (patient CV) for $\mathrm{P}_{3}$ show either complete or partial resistance to Bbsl and Hinfl digestion, respectively. The PCR products (sizes, in bp, shown on the left) from patients in which the consensus exon 8 sequence is present (patients DC, CS, and GS) are digested completely by Bbsl and Hinfl. Genotypes are: homozygous consensus sequence $(+/+)$, heterozygous carriers of the single-nucleotide deletion $\left(\mathrm{P}_{2}\right)$ in exon $8\left(+/ \mathrm{P}_{2}\right)$, homozygous carriers of $\mathrm{P}_{3}\left(\mathrm{P}_{3} / \mathrm{P}_{3}\right)$, and heterozygous carriers of $\mathrm{P}_{3}\left(+/ \mathrm{P}_{3}\right)$.

terone in the proband's serum $(9.2 \%)$ is very much higher than in a normal control (Figure 4c), or in normal women $(1.4 \%)$ of similar age with a mean serum SHBG concentration of $47 \mathrm{nM}$ (33). As a result, the concentration of free testosterone $(99 \mathrm{pM})$ in the proband's serum far exceeds the female reference range (6-22 pM) based on an average percentage free testosterone of $1.5 \%$, and this undoubtedly accounts for her hyperandrogenic symptoms. The amount of non-SHBG-bound testosterone (i.e., albumin-bound and free) in the proband's serum (697 pM) was also measured in an independent assay (28) and also great- ly exceeds the normal reference range (62-236 pM). Moreover, when compared with the total testosterone concentration in the proband's serum $(1.1 \mathrm{nM})$, these results confirm our estimates of the distribution of testosterone in the proband's serum: $9.2 \%$ free, $58.4 \%$ albumin-bound, and 32.4\% SHBG-bound.

Production and secretion of SHBG variants encoded by the proband's SHBG alleles. To determine whether the very low SHBG levels in serum samples from the proband are due to a defect in the production or secretion of the SHBG $\mathrm{P} 156 \mathrm{~L}$ variant and/or the carboxy-terminally truncated SHBG, we first expressed the variant alleles as cDNAs in $\mathrm{CHO}$ cells and performed a Western blot analysis of the resulting culture medium and transfected cell extracts. This indicated that the SHBG P156L variant is produced and secreted by CHO cells (Figure 5a, lanes 1 and 2), and that its steroid-binding characteristics are normal (Figure $5 \mathrm{~b}$ ). However, the relative abundance of the two major electrophoretic isoforms associated with SHBG P156L and normal SHBG differ (Figure 5a, lane 1 vs. lane 2), with the low-mobility isoform being predominant in SHBG P156L. A similar pattern is also seen when comparing the partially glycosylated monomers of SHBG P156L and SHBG in the insoluble cell extracts (Figure 5a, lane 7 vs. lane 8). By contrast, expression of a cDNA containing the single-nucleotide deletion found
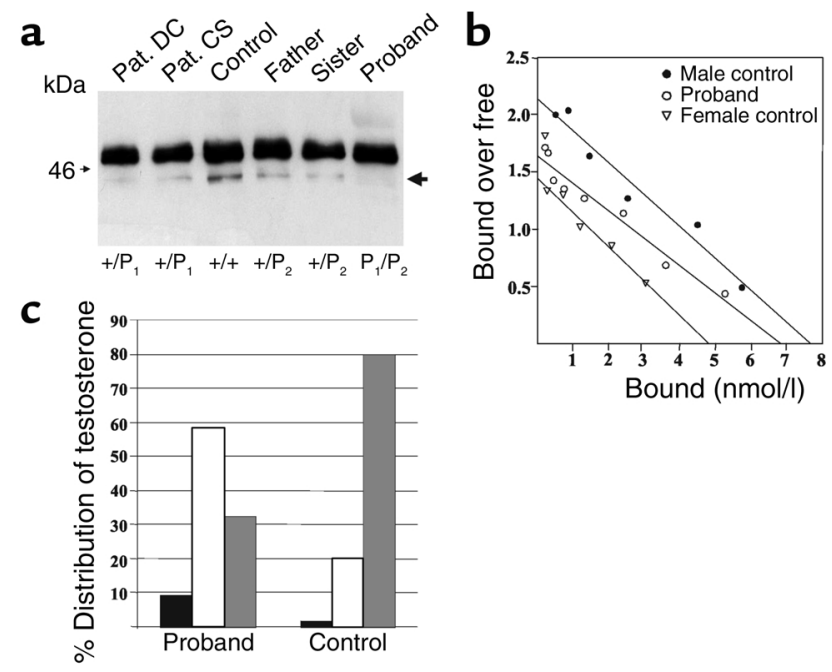

\section{Figure 4}

Biochemical properties of SHBG in serum from the proband and her family members, and from a normal female control and two patients (patients DC and CS) identified as heterozygous carriers of the Mspl polymorphism $\left(\mathrm{P}_{1}\right)$. (a) Western blot of SHBG immunoprecipitated from serum samples. Genotypes are: homozygous consensus sequence $(+/+)$, heterozygous carriers of the Mspl SNP $\left(+/ \mathrm{P}_{1}\right)$, heterozygous carriers of single-nucleotide deletion in exon $8\left(+/ P_{2}\right)$, and proband heterozygous for $\mathrm{P}_{1}$ and $\mathrm{P}_{2}\left(\mathrm{P}_{1} / \mathrm{P}_{2}\right)$. (b) Scatchard plots obtained for SHBG isolated from the proband $\left(K_{d}=4.1 \mathrm{nM}\right)$, and from male $\left(K_{\mathrm{d}}=3.6 \mathrm{nM}\right)$ and female $\left(K_{\mathrm{d}}=3.3 \mathrm{nM}\right)$ controls, using $\left[{ }^{3} \mathrm{H}\right] \mathrm{DHT}$ as labeled ligand (see Methods for details). (c) Distribution of testosterone between the SHBG-bound (gray bars), albuminbound (white bars), and non-protein-bound, or "free" (black bars), fractions in serum from the proband and a normal female control. 

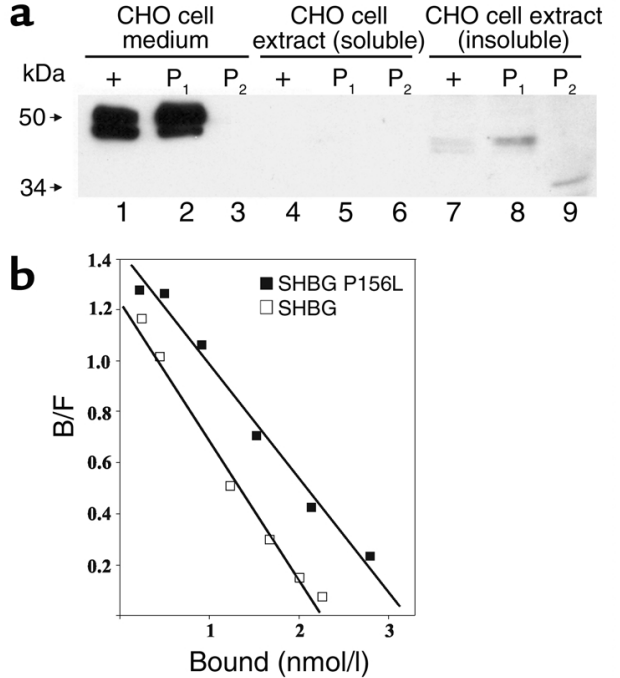

\section{Figure 5}

Comparison of the electrophoretic properties and steroid-binding characteristics of the products of the consensus and variant human SHBG cDNA sequences expressed in $\mathrm{CHO}$ cells. (a) Western blot of culture media and cell extracts from $\mathrm{CHO}$ cells transfected for stable expression of consensus $(+)$ and variant $\left(\mathrm{P}_{1}\right.$ or $\left.\mathrm{P}_{2}\right)$ SHBG cDNA sequences. $\mathrm{P}_{1}$, the $\mathrm{Mspl}$ polymorphism; $\mathrm{P}_{2}$, the single-nucleotide deletion in exon 8. Lanes are numbered under the blot. (b) Scatchard plots for human SHBG $\left(K_{d}=1.8 \mathrm{nM}\right)$ and the SHBG P156L variant $\left(K_{\mathrm{d}}=2.2 \mathrm{nM}\right)$ produced by $\mathrm{CHO}$ cells.

in exon 8 resulted in no SHBG in the culture medium (Figure 5a, lane 3), and only trace amounts of an approximately $34-\mathrm{kDa}$ immunoreactive truncation product within the insoluble extract of the transfected cells (Figure 5a, lane 9). In addition, since there was no evidence of a truncated form of SHBG on the Western blot of SHBG isolated from the proband's serum (Figure 4a), the very small amount of SHBG in the proband's serum must represent the product of the allele encoding the SHBG P156L variant.

Effect of the MspI polymorphism on SHBG expression. Apart from the MspI polymorphism $\left(\mathrm{P}_{1}\right)$, the allele encoding the SHBG P156L variant showed no other deviations from the consensus $S H B G$ sequence that might contribute to a reduction in its expression. This sequence analysis also included the 800-bp proximal promoter responsible for human SHBG expression in the liver (12). Since the MspI polymorphism is located close to the exon 4 /intron 4 boundary, we performed an experiment to determine whether it influences the splicing or relative abundance of SHBG transcripts, or their translation in hepatocytederived cell lines. This was done by introducing the sequences of the $\mathrm{P}_{1}$ variant or consensus $S H B G$ into BW-1 mouse hepatoma cells and human HepG2 hepatoblastoma cells. These genomic fragments span the translation start site for the SHBG precursor polypeptide and the polyadenylation sequence within exon 8 of human $\operatorname{SHBG}(9,12)$ and were expressed constitutively under the control of a CMV promoter. RT-PCR and Northern blot analysis of SHBG mRNA from stable transformants obtained after neomycin selection indicated that cells producing SHBG P156L contained only appropriately spliced $S H B G$ transcripts, as did cells expressing the consensus $S H B G$ sequences (Figure 6a). The relative abundance of $M s p$ I variant and consensus $S H B G$ transcripts in these cells was also essentially identical, but a Western blot indicated that the variant sequence consistently resulted in less SHBG P156L in the culture medium of these cells (data not shown). As in the case of the SHBG produced by $\mathrm{CHO}$ cells (Figure 5a), the electrophoretic isoform with reduced mobility associated with the SHBG P156L variant was always relatively more abundant than that in the normal protein, irrespective of the liver cell line in which it is expressed (data not shown). This is also consistent with the unique electrophoretic profile of the SHBG isolated from the proband's serum (Figure 4a), and these data indicated that the SHBG P156L variant is glycosylated abnormally and that its production and/or secretion might be reduced when compared with the
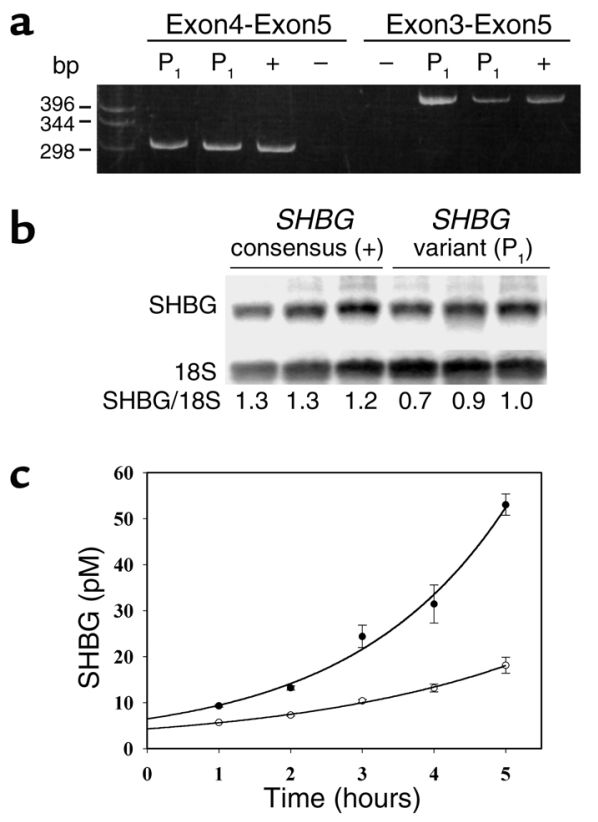

Figure 6

Expression of the Mspl variant SHBG allele $\left(\mathrm{P}_{1}\right)$ and the consensus $S H B G$ allele $(+)$ under the control of a CMV promoter in mouse BW-1 cells. (a) Ethidium bromide-stained polyacrylamide electrophoresis gel of RT-PCR amplification products obtained using primers that span the splice junctions between exons 4-5 and exons 3-5. Positions of DNA size markers are shown on the left. "-" indicates water control for PCR. (b) Northern blot of RNA extracted from replicate transfections of BW-1 cells with the expression vector ( $p R c / C M V)$ containing either the SHBG consensus or variant $\left(P_{1}\right)$ genomic sequences. The blot was probed with a human $\mathrm{SHBC}$ cDNA, and a cDNA for 18S RNA as a control for loading and transfer efficiency (30). Signals obtained using a Molecular Dynamics Phosphorlmager were quantified using ImageQuant software (Sunnyvale, California, USA), and SHBG/18S ratios are shown below the Northern blots. (c) Rate of SHBG secretion by near-confluent cultures containing similar amounts of SHBG transcripts, as shown in b. Data points are the means \pm SEM of SHBG levels in culture medium from replicate cultures of cells $(n=3)$ expressing the consensus $S H B G$ allele (filled circles) or the Mspl variant allele (open circles). 
SHBG produced by the consensus $S H B G$ allele. We confirmed this by comparing the secretion rate of the SHBG P156L variant and the normal SHBG protein from BW-1 cells transfected with either the variant or consensus human $S H B G$ sequences under the control of a CMV promoter. The relative abundance of $S H B G$ transcripts in these cells was similar (Figure $6 \mathrm{~b}$ ), while the amount of SHBG P156L in the culture medium, and the rate at which it accumulated, were clearly much reduced when compared with the SHBG produced by the consensus $S H B G$ sequence (Figure 6c).

\section{Discussion}

We have identified sequence variations within the coding regions of separate $S H B G$ alleles from a female patient who presented with severe hyperandrogenism during pregnancy. The level of SHBG in a blood sample taken 2 years after her pregnancy was exceptionally low, and we suspected a genetic abnormality might account for this. In an earlier report of a familial SHBG deficiency (7), the proband also suffered from symptoms (hirsutism and amenorrhea) consistent with androgen excess and ovarian dysfunction. Although the sensitivity of the assay used to measure serum SHBG in this previous report was only $10 \mathrm{nM}$ (7), SHBG was also undetectable in serum from her sister during mid-gestation (7), at a time when serum SHBG levels are usually in excess of $100 \mathrm{nM}$ (1). However, serum SHBG levels below $10 \mathrm{nM}$ are occasionally detected in patient samples using more sensitive immunoassay techniques, and it is therefore unclear whether the subjects investigated in this earlier report actually suffered from a total lack of SHBG.

The serum SHBG levels in the proband we identified were remarkably low, despite the fact that she had a normal body mass index and was under an estrogenic/ antiandrogenic treatment, which normally increases serum SHBG levels markedly. It is therefore likely that plasma SHBG levels must have also been very low during her pregnancy, and this would explain the severe hyperandrogenic symptoms she experienced at that time. What is also remarkable is that the twin girls she delivered showed no signs of virilization at birth. However, given the unusual maternal hormonal environment under which these infants developed, and the effects that exposures to endocrine active substances may have on health issues during later life (34), clinical monitoring of such infants is advisable.

The discovery of two novel mutations in the coding regions of separate SHBG alleles within a single patient is remarkable. The mendelian transmission of the two variant SHBG alleles within the proband's family is unambiguous, and she inherited the allele containing a deletion in exon 8 from her father, as did her sister. Her father's serum SHBG level $(52 \mathrm{nmol} / \mathrm{l})$ was within the normal reference range for men (9-54 $\mathrm{nmol} / \mathrm{l})$, but he was under treatment for hypertension and we cannot exclude the possibility that his medication influences SHBG production or its plasma half-life. Her sister's serum SHBG level (30 nmol/l) was only just within the normal female reference range, but at the time of blood sampling she was taking an oral contraceptive formulation that increases serum SHBG levels (35). This would be consistent with our findings that a cDNA representing the variant allele she inherited from her father is incapable of producing SHBG in CHO cells. It is also in line with previous observations that carboxy-terminal truncation mutants of rat $\mathrm{ABP}$ are not secreted when expressed in mammalian cells (18). However, mutant mRNAs that contain a frame-shift are known to undergo rapid degradation via a nonsense-mediated decay pathway (36), and this could also account for a lack of SHBG production from the proband's paternal $S H B G$ allele. Although we have only detected this particular variant $S H B G$ allele in the proband's immediate family, it is a null allele, and it will be important to screen other populations to determine whether individuals homozygous for this allele exist.

The proband must have inherited the allele containing the MspI polymorphism from her mother, who died prematurely due to unfortunate circumstances. There are $15 \mathrm{MspI}$ sites in human SHBG (GenBank accession no. M31651), and the mutation we have identified most likely accounts for an MspI restriction fragment length polymorphism (RFLP) that has an allele frequency of 0.04 in the French-Canadian population (37). No link has been made between this RFLP and serum SHBG levels, and no homozygotes have been reported, but the size of this RFLP is consistent with a polymorphism in the MspI site at the exon 4/intron 4 boundary. It is also of interest that many French-Canadians originate from the same region in France as does the proband. Given the prevalence of this MspI RFLP in the French-Canadian population, it will be important to confirm that this RFLP is due to the MspI polymorphism we have identified, because this may reveal homozygous carriers and provide more information about the clinical phenotype associated with this SNP.

The most intriguing question we faced is why the proband's serum SHBG levels were much lower than we expected given that the allele containing the MspI polymorphism appears to be expressed relatively normally when examined as a transgene in vitro. If we assume that the allele containing the deletion in exon 8 fails to contribute to the formation of plasma SHBG, as our expression studies imply, we would have expected to find at least $10 \mathrm{nM}$ of the SHBG P156L variant in the proband's serum unless its production is abnormally low. We therefore first examined the possibility that the MspI polymorphism in some way interferes with the processing of nascent $S H B G$ transcripts due to its proximity to an exon-intron consensus splice site or reduces the accumulation of $S H B G$ transcripts within cells. The results of our experimentsindicate that neither of these possibilities explain why the proband's serum SHBG levels are about an order of magnitude lower than expected. 
One consistent observation we made is that the relative abundance of the electrophoretic isoforms associated with the SHBG P156L variant differs from that of those associated with normal SHBG, and this can be attributed to differences in the utilization of the two sites for $\mathrm{N}$-glycosylation within the carboxy-terminal domain of the molecule (32). Since this amino acid substitution occurs within the amino-terminal LG-like domain of SHBG, it must somehow exert a long-range effect on posttranslational modifications within the carboxy-terminal region of the molecule; this is feasible, since we have observed similar effects when other amino acids are substituted within the amino-terminus of SHBG (16). Thus, the P156L substitution appears to influence the degree of N-glycosylation of SHBG, due presumably to subtle differences in the folding and posttranslational processing of the nascent polypeptide.

The P156L substitution occurs within a phylogenetically conserved region of SHBG (19) adjacent to residues located within the homodimer interface (16). It also lies within an inter- $\beta$ strand loop region that contains a coordination ligand (A160) for the calcium atom that plays an important role in maintaining the structural stability of the SHBG homodimer $(15,27)$. The substitution of a highly conserved proline at position 156 (19) might therefore be expected to influence the secondary structure of SHBG and might explain subtle differences in its posttranslational modification. It might also influence the rate of SHBG production/secretion or clearance from the blood circulation. Our observation that the SHBG P156L variant is secreted at a much reduced rate compared with that of normal SHBG from mouse liver cells, despite the fact that the levels of transcripts encoding them are similar in these cells, suggests that the variant protein is not processed efficiently during secretion. This is also consistent with the observation that greater amounts of immunoreactive SHBG are detected in the insoluble fraction of $\mathrm{CHO}$ cells expressing the SHBG P156L variant compared with the wild-type protein.

The genetic screening methods reported here will help identify individuals in whom genetic variants contribute to low serum SHBG levels, as opposed to others in whom serum SHBG levels are reduced because of some hormonal or metabolic factor that regulates SHBG production or metabolism. Our studies so far have focused on clinical disorders affecting women, and it will be important to determine whether the genetic variations we have identified in $S H B G$ are also linked to clinical conditions in men. In particular, it will be important to determine whether they are associated with reproductive disorders in men, because SHBG is expressed in the human testis and may play a role in spermatogenesis $(9,38)$. Finally, the use of these genetic screening methods should enable us to define more accurately how serum SHBG levels might be associated with other diseases. In addition, the presence of abnormally low plasma levels of SHBG in individuals who carry the $S H B G$ variants described in this report will undoubtedly lead to increased exposures to free testosterone and estradiol throughout their lifetimes and may contribute to the etiology of sex steroiddependent cancers of the prostate and breast.

\section{Acknowledgments}

We thank Denise Power and Myriam Richard for secretarial assistance, and David Rodenhiser for his critical review of the manuscript. We are also indebted to Allen Grolla, Christine Baret, and Claude Ducros for their excellent technical assistance, and Henri Déchaud for the serum testosterone analyses. This work was supported by the Canadian Institutes of Health Research (K.N. Hogeveen and G.L. Hammond) and la Fondation de France (P. Cousin and M. Pugeat). P. Cousin was supported by a postdoctoral fellowship from La Ligue National contre le Cancer.

1. Anderson, D.C. 1974. Sex-hormone-binding globulin. Clin. Endocrinol. (Oxf.) 3:69-96.

2. Hammond, G.L. 1990. Molecular properties of corticosteroid binding globulin and the sex-steroid binding proteins. Endocr. Rev. 11:65-79.

3. Siiteri, P.K., et al. 1982. The serum transport of steroid hormones. Recent Prog. Horm. Res. 38:457-510.

4. Selby, C. 1990. Sex hormone binding globulin: origin, function and clinical significance. Ann. Clin. Biochem. 27:532-541.

5. Lindstedt, G., et al. 1991. Low sex-hormone-binding globulin concentration as independent risk factor for development of NIDDM. 12-year follow-up of population study of women in Gothenborg Sweden. Diabetes. 40:123-128.

6. Lapidus, L., Lindstedt, G., Lundberg, P.-A., Bengtsson, C., and Gredmark, T. 1986. Concentrations of sex-hormone binding globulin and corticosteroid binding globulin in serum in relation to cardiovascular risk factors and to 12-year incidence of cardiovascular disease and overall mortality in postmenopausal women. Clin. Chem. 32:146-152.

7. Ahrentsen, O.D., Jensen, H.K., and Johnsen, S.G. 1982. Sex-hormonebinding globulin deficiency. Lancet. 2:377.

8. Meikle, A.W., Stanish, W.M., Taylor, N., Edwards, C.Q., and Bishop, C.T. 1982. Familial effects on plasma sex-steroid content in man: testosterone, estradiol and sex-hormone-binding globulin. Metabolism. 31:6-9.

9. Hammond, G.L., Underhill, D.A., Rykse, H.M., and Smith, C.L. 1989. The human sex hormone-binding globulin gene contains exons for androgen-binding protein and two other testicular messenger RNAs. Mol. Endocrinol. 3:1869-1876.

10. Bérubé, D., Seralini, G.-E., Gagné, R., and Hammond, G.L. 1990. Localization of the human sex hormone-binding globulin gene (SHBG) to the short arm of chromosome 17 (17p12-p13). Cytogenet. Cell Genet. 54:65-67

11. Khan, M.S., Knowles, B.B., Aden, D.P., and Rosner, W. 1981. Secretion of testosterone-estradiol-binding globulin by a human hepatoma-derived cell line. J. Clin. Endocrinol. Metab. 53:448-449.

12. Jänne, M., Deol, H.K., Power, S.G.A., Yee, S.-P., and Hammond, G.L. 1998. Human sex hormone-binding globulin gene expression in transgenic mice. Mol. Endocrinol. 12:123-136.

13. Rosner, W., Aden, D.P., and Khan, M.S. 1984. Hormonal influences on the secretion of steroid-binding proteins by a human hepatoma-derived cell line. J. Clin. Endocrinol. Metab. 59:806-808.

14. Plymate, S.R., Matej, L.A., Jones, R.E., and Friedl, K.E. 1988. Inhibition of sex hormone-binding globulin production in the human hepatoma (Hep G2) cell line by insulin and prolactin. J. Clin. Endocrinol. Metab. 67:460-464.

15. Grishkovskaya, I., et al. 2000. Crystal structure of human sex hormonebinding globulin: steroid transport by a laminin G-like domain. EMBO J. 19:504-512.

16. Avvakumov, G.V., Grishkovskaya, I., Muller, Y.A., and Hammond, G.L. 2001. Resolution of the human sex hormone-binding globulin dimer interface and evidence for two steroid-binding sites per homodimer. J. Biol. Chem. 276:34453-34457.

17. Avvakumov, G.V., Muller, Y.A., and Hammond, G.L. 2000. Steroid-binding specificity of human sex hormone-binding globulin is influenced by occupancy of a zinc-binding site. J. Biol. Chem. 275:25920-25925.

18. Joseph, D.R., and Lawrence, W. 1993. Mutagenesis of essential functional residues of rat androgen-binding protein/sex hormone-binding globulin. Mol. Endocrinol. 7:488-496.

19. Hammond, G.L. 1993. Extracellular steroid-binding proteins. In Steroid hormone action: frontiers in molecular biology. M.G. Parker, editor. IRL Press. Oxford, United Kingdom. 1-25. 
20. Power, S.G.A., et al. 1992. Molecular analyses of a human sex hormonebinding globulin variant: evidence for an additional carbohydrate chain J. Clin. Endocrinol. Metab. 75:1066-1070.

21. Cousin, P., Déchaud, H., Grenot, C., Lejeune, H., and Pugeat, M. 1998. Human variant sex hormone-binding globulin (SHBG) with an additional carbohydrate chain has a reduced clearance rate in rabbit. J. Clin. Endocrinol. Metab. 83:235-240.

22. Hogeveen, K.N., Talikka, M., and Hammond, G.L. 2001. Human sex hormone-binding globulin promoter activity is influenced by a (TAAAA), repeat element within an Alu sequence. J. Biol. Chem. 276:36383-36390.

23. Ferriman, D., and Gallwey, J.D. 1961. Clinical assessment of body hair growth in women. J. Clin. Endocrinol. Metab. 21:1440-1447.

24. Nisula, B.C., and Dunn, J.F. 1979. Measurement of the testosterone binding parameters for both testosterone-estradiol binding globulin and albumin in individual serum samples. Steroids. 34:771-791.

25. Hammond, G.L., and Lähteenmäki, P.L. 1983. A versatile method for the determination of serum cortisol binding globulin and sex hormone binding globulin binding capacities. Clin. Chim. Acta. 132:101-110.

26. Niemi, S., Mäentausta, O., Bolton, N.J., and Hammond, G.L. 1988. Timeresolved immunofluorometric assay of human sex hormone binding globulin (SHBG). Clin. Chem. 34:63-66.

27. Bocchinfuso, W.P., and Hammond, G.L. 1994. Steroid-binding and dimerization domains of human sex hormone-binding globulin partially overlap: steroids and $\mathrm{Ca}^{2+}$ stabilize dimer formation. Biochemistry. 33:10622-10629.

28. Déchaud, H., Lejeune, H., Garoscio-Cholet, M., Mallein, R., and Pugeat, M. 1989. Radioimmunoassay of testosterone not bound to sex-steroidbinding protein in plasma. Clin. Chem. 35:1609-1614.

29. Szpirer, C., and Szpirer, J. 1975. A mouse hepatoma cell line which secretes several serum proteins including albumin and alpha-foetoprotein. Differentiation. 4:85-91.
30. Bocchinfuso, W.P., Warmels-Rodenhiser, S., and Hammond, G.L. 1991. Expression and differential glycosylation of human sex hormone-binding globulin by mammalian cell lines. Mol. Endocrinol. 5:1723-1729.

31. Gershagen, S., Lundwall, A., and Fernlund, P. 1989. Characterization of the human sex hormone binding globulin (SHBG) gene and demonstration of two transcripts in both liver and testis. Nucleic Acids. Res. 17:9245-9258.

32. Bocchinfuso, W.P., Ma, K.-L., Lee, W.M., Warmels-Rodenhiser, S., and Hammond, G.L. 1992. Selective removal of glycosylation sites from sex hormone-binding globulin by site-directed mutagenesis. Endocrinology. 131:2331-2336.

33. Hammond, G.L., Langley, M.S., Robinson, P.A., Nummi, S., and Lund, L. 1984. Serum steroid binding protein concentrations, distribution of progestogens, and bioavailability of testosterone during treatment with contraceptives containing desogestrel or levonorgestrel. Fertil. Steril. 42:44-51.

34. McLachlan, J.A. 2001. Environmental signaling: what embryos and evolution teach us about endocrine disrupting chemicals. Endocr. Rev. 22:319-341.

35. Kuhl, H., Jung-Hoffmann, C., and Wiegratz, I. 1995. Gestodene-containing contraceptives. Clin. Obstet. Gynecol. 38:829-840.

36. Byers, P. H. 2002. Killing the messenger: new insights into nonsensemediated mRNA decay. J. Clin. Invest. 109:3-6.

37. Vohl, M.-C., Dionne, F.T., Dériaz, O., Chagnon, M., and Bouchard, C. 1994. Detection of a MspI restriction fragment length polymorphism for the human sex hormone-binding globulin (SHBG) gene. Hum. Genet. 93:84.

38. Joseph, D.R. 1994. Structure, function, and regulation of androgenbinding protein/sex hormone-binding globulin. Vitam. Horm. 49:197-280. 